\title{
Doubly warped product submanifolds of a Riemannian manifold of nearly quasi-constant curvature
}

\author{
Mehraj Ahmad Lone, Mohamd Saleem Lone and Mohammad Hasan Shahid
}

\begin{abstract}
In the present paper, we form a sharp inequality for a doubly warped product submanifold of a Riemannian manifold of nearly quasi-constant curvature.
\end{abstract}

Keywords. Chen inequality, doubly warped product manifold, nearly quasi-constant curvature tensor

\section{Introduction}

In [7] B.-Y Chen and K. Yano introduced the notion of quasi-constant curvature. A Riemannian manifold $(\mathcal{M}, g)$ is called a Riemannian manifold of quasi-constant curvature if its curvature tensor $R$ satisfies the condition

$$
\begin{aligned}
R(X, Y, Z, W)= & a[g(Y, Z) g(X, W)-g(X, Z) g(Y, W)] \\
& +b[g(X, W) A(Y) A(Z)-g(X, Z) A(Y) A(W) \\
& +g(Y, Z) A(X) A(W)-g(Y, W) A(X) A(Z)],
\end{aligned}
$$

where $a, b$ are scalar functions and $A$ is a 1 -form given by

$$
g(X, P)=A(X),
$$

$P$ is a fixed unit vector field. It is straightforward to see that if $b=0$, then $(\mathcal{M}, g)$ reduces to a Riemannian manifold of constant curvature.

For $n>2$, a non-flat Riemannian manifold $\left(\mathcal{M}^{n}, g\right)$ is said to be a quasi-Einstein manifold if its Ricci tensor satisfies the condition

$$
S(X, Y)=a g(X, Y)+b A(X) A(Y),
$$

where $a, b$ are scalar functions and $A$ is 1 -form acting same as above. It can be easily verified that every Riemannian manifold of quasi-constant curvature is a quasi-Einstein manifold.

In 2009, the notion of quasi-constant curvature was generalized to nearly quasi-constant by A. K. Gazi and U. C. De (see [8]). It is a Riemannian manifold whose curvature tensor satisfies

$$
R(X, Y, Z, W)=p[g(Y, Z) g(X, W)-g(X, Z) g(Y, W)]
$$

Received date: May 28, 2020; Published online: April 13, 2021.

2010 Mathematics Subject Classification. 53C05, 53C40.

Corresponding author: Mehraj Ahmad Lone. 


$$
\begin{aligned}
& +q[g(X, W) B(Y, Z)-g(X, Z) B(Y, W) \\
& +g(Y, Z) B(X, W)-g(Y, W) B(X, Z)],
\end{aligned}
$$

where $a, b$ are scalar functions and $B$ is a non-vanishing $(0,2)$ type symmetric tensor.

For $n>2$, a non-flat Riemannian manifold $\left(\mathcal{M}^{n}, g\right)$ is said to be a nearly quasi-Einstein manifold if its Ricci tensor satisfy

$$
S(X, Y)=a g(X, Y)+b B(X, Y) .
$$

It can be easily verified that every Riemannian manifold of nearly quasi-constant curvature is a nearly quasi-Einstein manifold.

We know that the outer product of two convariant vectors is a covariant $(0,2)$ tensor, but not conversely true always. Hence nearly quasi-constant Riemannian manifolds act as a bigger class of Riemannian manifolds in the sense that every Riemannian manifold of quasi-constant curvature is nearly quasi-constant Riemannian manifold, but there are plenty of examples where the converse is not true.

Example 1. ([8]) Let $\left(\mathbb{R}^{4}, g\right)$ be a Riemannian manifold with the metric $g$ defined as follows

$$
d s^{2}=\left(x^{4}\right)^{\frac{4}{5}}\left[\left(d x^{1}\right)^{2}+\left(d x^{2}\right)^{2}+\left(d x^{3}\right)^{2}\right]+\left(d x^{4}\right)^{2} .
$$

This is a nearly quasi-constant Riemannian manifold but not a quasi-constant Riemannian manifold.

In an attempt to construct Riemannian manifolds with negative sectional curvature, O' Neill and Bishop introduced the notion of singly warped products (see [3]). The warped product manifold model has plenty of applications in relativity. In [1], Beem, Ehrlich and Powell showed the exact solutions to Einstein's field equation are expressible in terms of Lorentzian warped products. For more applications, see $[2,6]$.

Definition 1. Let $\left(\mathcal{M}_{1}, g_{1}\right)$ and $\left(\mathcal{M}_{2}, g_{2}\right)$ be two Riemannian manifolds, the warped product $\mathcal{M}=\mathcal{M}_{1} \times{ }_{\alpha} \mathcal{M}_{2}$ is the product manifold equipped with the metric

$$
g=\pi_{1}^{*}\left(g_{1}\right)+\left(\alpha \circ \pi_{1}\right)^{2} \pi_{2}^{*}\left(g_{2}\right),
$$

where $\alpha: \mathcal{M}_{1} \rightarrow(0, \infty)$ is a smooth function on $\mathcal{M}_{1}, \pi_{i}: \mathcal{M}_{1} \times \mathcal{M}_{2} \rightarrow \mathcal{M}_{i}, i=1,2$ are the projections and $*$ is the pullback. The Riemannian manifold $\left(\mathcal{M}_{1}, g_{1}\right)$ is called as the base, $\left(\mathcal{M}_{2}, g_{2}\right)$ is called as fibre and $\alpha$ is called as the warping function of the warped product.

A very prominent example of warped product manifold is a generalized Robertson-Walker space-time, which is a Lorentzian warped product of the form $\mathcal{M}=(a, b) \times{ }_{\alpha} \mathcal{N}$, where $(a, b)$ is an open interval, $\mathcal{N}$ is a three dimensional space form and the metric on $\mathcal{M}$ is given by $g=-d t^{2}+\alpha^{2} g_{\mathcal{N}}($ cf. $[2,9])$.

The notion of doubly warped manifold can be considered as a natural generalization of singly warped product manifold.

Definition 2. Let $\left(\mathcal{M}_{1}, g_{1}\right)$ and $\left(\mathcal{M}_{2}, g_{2}\right)$ be two Riemannian manifolds, the doubly warped product $\mathcal{M}={ }_{\alpha_{2}} \mathcal{M}_{1} \times{ }_{\alpha_{1}} \mathcal{M}_{2}$ is the product manifold equipped with the metric

$$
g=\left(\alpha_{2} \circ \pi_{2}\right)^{2} \pi_{1}^{*}\left(g_{1}\right)+\left(\alpha_{1} \circ \pi_{1}\right)^{2} \pi_{2}^{*}\left(g_{2}\right),
$$


where $\alpha_{i}: \mathcal{M}_{i} \rightarrow(0, \infty)$ is a smooth function on $\mathcal{M}_{i}, \pi_{i}: \mathcal{M}_{1} \times \mathcal{M}_{2} \rightarrow \mathcal{M}_{i}, i=1,2$ are the usual projections and $*$ is the pullback. If one of $\alpha_{i}=1$, but not both, then we get warped product manifold. If both $\alpha_{i}=1$, we get a Riemannian product manifold. If neither of $\alpha_{i}$ is constant, we get a non-trivial doubly product manifold (see [12]).

The structure of the paper is as following. In section 2, we compile the basic definitions and all the prerequisites needed afterwards. In section 3 , we prove our main result.

\section{Preliminaries}

Let $\mathcal{M}$ be an $n$-dimensional Riemannian submanifold of a Riemannian $m$-dimensional manifold $\mathcal{N}$ and let $\nabla$ and $\hat{\nabla}$ be the Levi-Civita connection of $\mathcal{M}, \mathcal{N}$, respectively. Then the Gauss and Weingarten formula are given respectively by

$$
\begin{aligned}
\hat{\nabla}_{X} Y & =\nabla_{X} Y+h(X, Y) \\
\hat{\nabla}_{X} \xi & =-A_{\xi} X+\nabla_{X}^{\perp} \xi
\end{aligned}
$$

for all $X, Y \in \Gamma(T \mathcal{M})$ and $\xi \in \Gamma\left(T^{\perp} \mathcal{M}\right)$, where $\nabla^{\perp}$ is the normal connection, $A$ is the shape operator and $h$ is the second fundamental form and are related by the relation

$$
g(h(X, Y), \xi)=g\left(A_{\xi} X, Y\right) .
$$

The Gauss equation is given by

$$
\hat{R}(X, Y, Z, W)=R(X, Y, Z, W)-g(h(X, W), h(Y, Z)+g(h(X, Z), h(Y, W))
$$

for all $X, Y, Z, W \in \Gamma(T \mathcal{M})$, where $\hat{R}$ is the curvature tensor of $\mathcal{N}$ and $R$ is the induced curvature tensor on $\mathcal{M}$.

Let $\left\{e_{1}, e_{2}, \cdots, e_{n}\right\},\left\{e_{n+1}, \cdots, e_{m}\right\}$ be orthonormal basis of the tangent space $T_{p}(\mathcal{M})$ and $T_{p}^{\perp}(\mathcal{M})$, respectively. Then the mean curvature field is given by

$$
H=\frac{1}{n} \sum_{i=1}^{n} h\left(e_{i}, e_{i}\right)=\frac{1}{n} \sum_{r=n+1}^{m}\left(\sum_{i=1}^{n} h_{i i}^{r}\right) e_{r}, h_{i j}^{r}=g\left(h\left(e_{i}, e_{j}\right), e_{r}\right),
$$

$1 \leq i, j \leq n, n+1 \leq r \leq m$.

Suppose $\alpha$ is a differentiable function on $\mathcal{M}$, then the Laplacian $\Delta \alpha$ is defined as

$$
\Delta \alpha=\sum_{i=1}^{n}\left[\left(\nabla_{e_{i}} e_{i}\right) \alpha-e_{i} e_{i} \alpha\right]
$$

Let $\pi \subset T_{p} \mathcal{M}$ be a 2-plane section and $K(\pi)$ be the sectional curvature of $\mathcal{M}$. Then for an orthonomal basis $\left\{e_{1}, \cdots, e_{n}\right\}$ of the tangent space, the scalar curvature is defined as

$$
\tau=\sum_{1 \leq i<j \leq n} K\left(e_{i} \wedge e_{j}\right)
$$

Now for a doubly warped product manifold, assuming $\mathcal{D}_{1}, \mathcal{D}_{2}$ the distributions obtained from the vectors tangent to leaves and fibres, respectively. Let $s:{ }_{\alpha_{2}} \mathcal{M}_{1} \times{ }_{\alpha_{1}} \mathcal{M}_{2} \rightarrow \mathcal{N}$ be an isometric immersion, then we have

$$
H_{i}=\frac{1}{n_{i}} \operatorname{tr}\left(h_{i}\right)
$$


the partial mean curvature, where $\operatorname{tr}\left(h_{i}\right)$ is the trace of $h$ restricted to $\mathcal{M}_{i}$ and $n_{i}=\operatorname{dim} \mathcal{M}_{i}$. The doubly warped product manifold is called as mixed totally geodesic if $h(X, Y)=0$ for any $X, Y$ tangent to $\mathcal{D}_{1}, \mathcal{D}_{2}$, respectively.

For a warped product submanifold of a Riemannian manifold of constant sectional curvature, B.-Y Chen proved the following:

Theorem 2.1. [5] Let $\mathcal{M}=\mathcal{M}_{1} \times{ }_{\alpha} \mathcal{M}_{2}$ be an n-dimensional warped product submanifold of a Riemannian manifold $\mathcal{N}(c)$. Then, we have

$$
\frac{\Delta \alpha}{\alpha} \leq \frac{n^{2}}{4 n_{2}}\|H\|^{2}+n_{1} c
$$

where $n_{i}=\operatorname{dim} \mathcal{M}_{i}, n=n_{1}+n_{2}$. The equality holds if and only if $\mathcal{M}$ is a mixed totally geodesic and $n_{1} H_{1}=n_{2} H_{2}, H_{i}$ is the partial mean curvature vectors, $i=1,2$.

Later in [10], A. Olteanu obtained a sharp inequality for a doubly warped product submanifold of an arbitrary Riemannian manifold. In [12], using the quasi-constant curvature tensor, S. Sular obtained a sharp inequality for a doubly warped product submanifold of a Riemannian manifold. Motivated by the above studies, we discuss a sharp inequality for a doubly warped product submanifold of a Riemannian manifold of nearly quasi-constant curvature.

We shall use the following Chen's lemma while proving our main result.

Lemma 2.1. [4] For $m \geq 2$ and $b_{1}, b_{2}, \cdots, b_{m}, \mu$ be reals, such that

$$
\left(\sum_{j=1}^{m} b_{i}\right)^{2}=(m-1)\left(\sum_{j=1}^{m} b_{i}^{2}+\mu\right) .
$$

Then $2 b_{1} b_{2} \geq \mu$, with equality if and only if $b_{1}+b_{2}=b_{3}=\cdots=b_{m}$.

\section{Doubly warped product submanifolds}

In this section, we derive a sharp relationship between a doubly warped product submanifold $\mathcal{M}={ }_{\alpha_{2}} \mathcal{M}_{1} \times{ }_{\alpha_{1}} \mathcal{M}_{2}$, its warping functions and the squared mean curvature.

Theorem 3.1. Let $\mathcal{M}={ }_{\alpha_{2}} \mathcal{M}_{1} \times{ }_{\alpha_{1}} \mathcal{M}_{2}$ be an n-dimensional doubly warped product submanifold of an $m$-dimensional Riemannian manifold $\mathcal{N}$. Then we have

$$
n_{2} \frac{\Delta_{1} \alpha_{1}}{\alpha_{1}}+n_{1} \frac{\Delta_{2} \alpha_{2}}{\alpha_{2}} \leq \frac{n^{2}}{4}\|H\|^{2}+n_{1} n_{2} p-q(n-1) \operatorname{tr}(B),
$$

where $n_{1}+n_{2}=n, n_{i}=\operatorname{dim} \mathcal{M}_{i}$ and $\Delta_{i}$ is the Laplacian of $\mathcal{M}_{i}$. The equality in (3.13) holds if and only if $\mathcal{M}$ is totally geodesic with $\operatorname{tr}\left(h_{1}\right)=\operatorname{tr}\left(h_{2}\right)$.

Proof. Let $\mathcal{M}$ be a doubly warped product submanifold of a Riemannian manifold $\mathcal{N}$ of nearly quasi-constant curvature. Then, we have

$$
\begin{aligned}
& \nabla_{X} Y=\nabla_{X}^{\mathcal{M}_{1}}-\frac{\alpha_{2}^{2}}{\alpha_{1}^{2}} g_{1}(X, Y) \nabla^{\mathcal{M}_{2}}\left(\ln \alpha_{2}\right) \\
& \nabla_{X} Z=Z\left(\ln \alpha_{2}\right) X+X\left(\ln \alpha_{1}\right) Z
\end{aligned}
$$


for any $X, Y \in \Gamma\left(T \mathcal{M}_{1}\right), Z \in \Gamma\left(T \mathcal{M}_{2}\right)$, where $\nabla^{\mathcal{M}_{i}}$ is the Levi-Civita connection of the Riemannian metric $g_{i}, i=1,2$.

The sectional curvature of the $\{X, Y\}$ plane is given by

$$
K(X \wedge Y)=\frac{1}{\alpha_{1}}\left[\left(\nabla_{X}^{\mathcal{M}_{1}} X\right) \alpha_{1}-X^{2} \alpha_{1}\right]+\frac{1}{\alpha_{2}}\left[\left(\nabla_{Y}^{\mathcal{M}_{1}} Y\right) \alpha_{2}-Y^{2} \alpha_{2}\right]
$$

Fix an orthonormal basis $\left\{e_{1} \cdots, e_{n_{1}} e_{n_{1}+1}, \cdots, e_{n}\right\}$, such that first $n_{1}$ tuples acts as basis of $T_{p} \mathcal{M}_{1}$ and the remaining of $T_{p} \mathcal{M}_{2}$ and $e_{n_{1}+1} \| H$, we get

$$
n_{2} \frac{\Delta_{1} \alpha_{1}}{\alpha_{1}}+n_{1} \frac{\Delta_{2} \alpha_{2}}{\alpha_{2}}=\sum_{1 \leq s_{1} \leq n_{1}<s_{2} \leq n} K\left(e_{s_{1}} \wedge e_{s_{2}}\right)
$$

for each $s_{2} \in\left\{n_{1}+1, \cdots, n\right\}$.

Using Gauss equation for $X=W=e_{i}$ and $Y=Z=e_{j}, i \neq j$, we have

$$
2 \tau=n^{2}\|H\|^{2}-\|h\|^{2}+2 q(n-1) \operatorname{tr}(B)+\left(n^{2}-n\right) p,
$$

where

$$
\|h\|^{2}=\sum_{i, j=1}^{n} g\left(h\left(e_{i}, e_{j}\right),\left(h\left(e_{i}, e_{j}\right)\right)\right.
$$

is the squared norm of the second fundamental form $h$ and $\tau$ is the scalar curvture.

We fix

$$
\epsilon=2 \tau-\frac{n^{2}}{2}\|H\|^{2}-\left(n^{2}-n\right) p-2 q(n-1) \operatorname{tr}(B) .
$$

Then, from (3.3) and (3.4), we get

$$
n^{2}\|H\|^{2}=2\left(\|h\|^{2}+\epsilon\right)
$$

For a suitable local orthonormal frame, the above relation can be written as

$$
\left(\sum_{i=1}^{n} h_{i i}^{n+1}\right)^{2}=2\left[\epsilon+\sum_{i=1}^{n}\left(h_{i i}^{n+1}\right)^{2}+\sum_{i \neq j}\left(h_{i j}^{n+1}\right)^{2}+\sum_{r=n+2}^{m} \sum_{i, j=1}^{n}\left(h_{i j}^{r}\right)^{2}\right] .
$$

Put $b_{1}=h_{11}^{n+1}, b_{2}=\sum_{i=2}^{n_{1}} h_{i i}^{n+1}$ and $b_{3}=\sum_{t=n_{1}+1}^{n} h_{t t}^{n+1}$, the previous equation is equivalent to

$$
\begin{aligned}
\left(\sum_{i=1}^{3} b_{i}\right)^{2}= & 2\left[\epsilon+\sum_{i=1}^{3} b_{i}^{2}+\sum_{1 \leq i \neq j \leq n}\left(h_{i j}^{n+1}\right)^{2}+\sum_{r=n+2}^{m} \sum_{i, j=1}^{n}\left(h_{i j}^{r}\right)^{2}\right. \\
& \left.-\sum_{2 \leq j \neq k \leq n_{1}} h_{j j}^{n+1} h_{k k}^{n+1}-\sum_{n+1 \leq s \neq t \leq n} h_{s s}^{n+1} h_{t t}^{n+1}\right] .
\end{aligned}
$$

For $b_{1}, b_{2}, b_{3}$, we see that, (3.6) satisfy lemma 2.1, implying

$$
\left(\sum_{i=1}^{3} b_{i}\right)^{2}=2\left(\sum_{i=1}^{3} b_{i}^{2}+\mu\right)
$$


where

$$
\begin{aligned}
\mu= & \epsilon+\sum_{1 \leq i \neq j \leq n}\left(h_{i j}^{n+1}\right)^{2}+\sum_{r=n+2}^{m} \sum_{i, j=1}^{n}\left(h_{i j}^{r}\right)^{2} \\
& -\sum_{2 \leq j \neq k \leq n_{1}} h_{j j}^{n+1} h_{k k}^{n+1}-\sum_{n+1 \leq s \neq t \leq n} h_{s s}^{n+1} h_{t t}^{n+1} .
\end{aligned}
$$

Then, we obtain $2 b_{1} b_{2} \geq \mu$, with equality if and only if $b_{1}+b_{2}=b_{3}$, or

$$
\begin{array}{r}
\sum_{1 \leq j<k \leq n_{1}} h_{j j}^{n+1} h_{k k}^{n+1}+\sum_{n+1 \leq s<t \leq n} h_{s s}^{n+1} h_{t t}^{n+1} \\
\geq \frac{\epsilon}{2}+\sum_{1 \leq \alpha<\beta \leq n}\left(h_{\alpha \beta}^{n+1}\right)^{2}+\frac{1}{2} \sum_{r=n+2}^{m} \sum_{\alpha, \beta=1}^{n}\left(h_{\alpha \beta}^{r}\right)^{2} .
\end{array}
$$

The equality holds if and only if

$$
\sum_{i=1}^{n_{1}} h_{i i}^{n+1}=\sum_{t=n_{1}+1}^{n} h_{t t}^{n+1} .
$$

Using Gauss equation again, we have

$$
\begin{aligned}
n_{2} \frac{\Delta_{1} \alpha_{1}}{\alpha_{1}}+n_{1} \frac{\Delta_{2} \alpha_{2}}{\alpha_{2}}= & \tau-\sum_{1 \leq j<k \leq n_{1}} K\left(e_{j} \wedge e_{k}\right)-\sum_{n_{1}+1 \leq s<t \leq n} K\left(e_{s} \wedge e_{t}\right) \\
= & \tau-\frac{1}{2} n_{1} p\left(n_{1}-1\right)-\sum_{r=n+1}^{m} \sum_{1 \leq j<k \leq n_{1}}\left(h_{j j}^{r} h_{k k}^{r}-\left(h_{j k}^{r}\right)^{2}\right) \\
& -q\left(n_{1}-1\right) \operatorname{tr}(B)-\frac{1}{2} n_{2} p\left(n_{2}-1\right) \\
& -\sum_{r=n+1}^{m} \sum_{n_{1}+1 \leq s<t \leq n_{1}}\left(h_{s s}^{r} h_{t t}^{r}-\left(h_{s t}^{r}\right)^{2}\right)-q\left(n_{2}-1\right) \operatorname{tr}(B) .
\end{aligned}
$$

Using (3.2), (3.7) and (3.9), we get

$$
\begin{aligned}
& n_{2} \frac{\Delta_{1} \alpha_{1}}{\alpha_{1}}+n_{1} \frac{\Delta_{2} \alpha_{2}}{\alpha_{2}} \leq \tau-\frac{1}{2} n p(n-1)+n_{1} n_{2} p-\frac{\epsilon}{2}-\frac{1}{2} \sum_{r=n+2}^{m} \sum_{\alpha, \beta}^{n}\left(h_{\alpha \beta}^{r}\right)^{2} \\
& +\sum_{r=n+2}^{m} \sum_{1 \leq j<k \leq n_{1}}\left(\left(h_{j k}^{r}\right)^{2}-h_{j j}^{r} h_{k k}^{r}\right)+\sum_{r=n+2}^{m} \sum_{n_{1}+1 \leq s<t \leq n_{1}}\left(\left(h_{s t}^{r}\right)^{2}-h_{s s}^{r} h_{t t}^{r}\right) \\
& -q\left(n_{1}-1\right) \operatorname{tr}(B)-q\left(n_{2}-1\right) \operatorname{tr}(B) . \\
& =\tau-\frac{1}{2} n p(n-1)+n_{1} n_{2} p-\frac{\epsilon}{2}-\sum_{r=n+1}^{m} \sum_{j=1}^{n_{1}} \sum_{t=n_{1}+1}^{n}\left(h_{j t}^{r}\right)^{2} \\
& -\frac{1}{2} \sum_{r=n+2}^{m}\left(\sum_{j=1}^{n_{1}} h_{j j}^{r}\right)^{2}-\frac{1}{2} \sum_{r=n+2}^{m}\left(\sum_{t=n_{1}+1}^{n} h_{t t}^{r}\right)^{2} \\
& -q\left(n_{1}-1\right) \operatorname{tr}(B)-q\left(n_{2}-1\right) \operatorname{tr}(B) \\
& \leq \tau-\frac{1}{2} n p(n-1)+n_{1} n_{2} p-\frac{\epsilon}{2}-q\left(n_{1}-1\right) \operatorname{tr}(B)-q\left(n_{2}-1\right) \operatorname{tr}(B)
\end{aligned}
$$




$$
=\frac{n^{2}}{4}\|H\|^{2}+n_{1} n_{2} p-q(n-1) \operatorname{tr}(B) .
$$

This proves our claim.

It is straightforward to check that the equality holds in (3.13), if and only if

$$
h_{j l}^{r}=0, n+1 \leq r \leq m
$$

and

$$
\sum_{i=1}^{n_{1}} h_{i i}^{r}=\sum_{l=n_{1}+1}^{n} h_{l l}^{r}=0
$$

where $1 \leq j \leq n_{1}, n_{1}+1 \leq l \leq n$ and $n+2 \leq r \leq m$.

From (3.10) vanishing of the second fundamental form of ${ }_{\alpha_{2}} \mathcal{M}_{1} \times{ }_{\alpha_{1}} \mathcal{M}_{2}$ in $\mathcal{N}$ implies $h\left(\mathcal{D}_{1}, \mathcal{D}_{2}\right)=$ $\{0\}$, or we can say that the immersion $s$ is totally geodesic. Again from (3.8) and (3.11), we see that

$$
\sum_{s_{1}=1}^{n_{1}} h\left(e_{s_{1}}, e_{s_{2}}\right)=\sum_{s_{2}=n_{1}+1}^{n} h\left(e_{s_{2}}, s_{s_{2}}\right)
$$

implying $\operatorname{tr}\left(h_{1}\right)=\operatorname{tr}\left(h_{2}\right)$.

Conversely assuming $\mathcal{N}$ is the required Riemannian manifold, such that $\mathcal{M}$ is totally geodesic with $\operatorname{tr}\left(h_{1}\right)=\operatorname{tr}\left(h_{2}\right)$, then the equality in (3.13) follows easily.

Corollary 3.2. Let $\mathcal{M}={ }_{\alpha_{2}} \mathcal{M}_{1} \times{ }_{\alpha_{1}} \mathcal{M}_{2}$ be a compact, orientable $n$-dimensional doubly warped product submanifold of an $m$-dimensional Riemannian manifold $\mathcal{N}$. Then we have

$$
\|H\|^{2} \geq \frac{4}{n^{2}}\left[q(n-1) \operatorname{tr}(B)-n_{1} n_{2} p\right] .
$$

Proof. Suppose $\mathcal{M}$ be a compact orientable Riemannian manifold without boundary satisfying (3.13), then we have

$$
n_{2} \frac{\Delta_{1} \alpha_{1}}{\alpha_{1}}+n_{1} \frac{\Delta_{2} \alpha_{2}}{\alpha_{2}} \leq \frac{n^{2}}{4}\|H\|^{2}+n_{1} n_{2} p-q(n-1) \operatorname{tr}(B) .
$$

Therefore, from the definition of volume element

$$
\int_{\mathcal{M}} \Delta_{i} \alpha_{i} d V=0, \quad i=1,2 .
$$

Thus, we get

$$
0 \leq \int_{\mathcal{M}}\left(\frac{n^{2}}{4}\|H\|^{2}+n_{1} n_{2} p-q(n-1) \operatorname{tr}(B)\right) d V=0
$$

Corollary 3.3. Let $\mathcal{M}={ }_{\alpha_{2}} \mathcal{M}_{1} \times{ }_{\alpha_{1}} \mathcal{M}_{2}$ be an $n$-dimensional doubly warped product submanifold of an $m$-dimensional Riemannian manifold $\mathcal{N}$ satisfying

$$
n_{2} \frac{\Delta_{1} \alpha_{1}}{\alpha_{1}}+n_{1} \frac{\Delta_{2} \alpha_{2}}{\alpha_{2}}>n_{1} n_{2} p-q(n-1) \operatorname{tr}(B),
$$

then $\mathcal{M}$ is non-minimal. 


\section{Acknowledgments}

We would like to thank the referees for careful reading of the paper and pointing out some errors which improved the paper.

\section{References}

[1] J. Beem, P. Ehrlich and T. G. Powell, Warped product manifolds in relativity, Selected Studies: A volume dedicated to the memory of Albert Einstein (1982), 4156.

[2] J. K. Beem, Global Lorentzian geometry, Routledge, 2017.

[3] R. L. Bishop and B. ONeill, Manifolds of negative curvature, Transactions of the American Mathematical Society 145 (1969), 149.

[4] B. Y. Chen, Some pinching and classification theorems for minimal submanifolds, Archiv der Mathematik 60 (1993), 568578.

[5] B. Y. Chen, On isometric minimal immersions from warped products into real space forms, Proceedings of the Edinburgh Mathematical Society 45 (2002), 579587.

[6] B. Y. Chen, Differential geometry of warped product manifolds and submanifolds, World Scientific Singapore, 2017.

[7] B. Y. Chen and K. Yano, Hypersurfaces of a conformally flat space, Tensor(NS) 26 (1972), 318322 .

[8] A. K. Gazi and U. C. De On the existence of nearly quasi-Einstein manifolds, Novi Sad J. Math 39 (2009), 111117.

[9] S. W. Hawking and G. F. R. Ellis, The large scale structure of space-time, vol. 1. Cambridge university press, 1973.

[10] A. Olteanu, A general inequality for doubly warped product submanifolds, Mathematical Journal of Okayama University 52 (2010), 133-142.

[11] S. Sular, Doubly warped product submanifolds of a Riemannian manifold of quasi-constant curvature, Annals of the Alexandru Ioan Cuza University- Mathematics 61 (2015), 235244.

[12] B. Unal, Doubly warped products [ph. d. thesis], University of Missouri Columbia (2000).

Mehraj Ahmad Lone National Institute of Technology Srinagar, J\&K, India

E-mail: mehrajlone@nitsri.ac.in, mehraj.jmi@gmail.com

Mohammad Saleem Lone International Centre for Theoretical Sciences, Tata Institute of Fundamental Research, 560089, Bengaluru, India.

E-mail: saleemraja2008@gmail.com

Mohammad Hasan Shahid New Delhi, India.
Department of Mathematics, Jamia Millia Islamia , 110025, 
E-mail: hasan_jmi@yahoo.com 